\title{
Edge caching strategy design and reward contract optimization for uAV-enabled mobile edge networks
}

\author{
Weidang Lu ${ }^{*}$ (D), Bin Yin ${ }^{1}$, Guoxiang Huang ${ }^{1}$ and Bo Li
}

\begin{abstract}
Edge caching is a promising technology to alleviate the burden on backhaul and improve the quality of experience (QoE) of users in unmanned aerial vehicle (UAV)-enabled mobile edge networks, which has played an important role in wireless communication systems. However, owing to the selfish nature and limited battery life of the user equipments (UEs), only a limited part of the caching resources will be shared by the UEs. To drive the UEs to share more caching resources to improve the social welfare of the UAV-enabled mobile edge network, in this paper, we jointly design an edge caching strategy and reward contract optimization scheme. Aiming to maximize the utility of the UAV, a joint edge caching and contract optimization problem is formulated. Firstly, a novel edge caching strategy is proposed based on the content popularity. Then, an optimal reward contract is designed by reducing the restrictions. Finally, the system performance of the designed caching strategy and reward contract is evaluated compared with two benchmark caching schemes with no incentives. Simulation results show that the proposed scheme performs better than the other two schemes in terms of the utilities of the UAV and UEs, which proves the efficiency of our proposed scheme.
\end{abstract}

Keywords: Edge caching, UAV communications, Contract design, Information asymmetry

\section{Introduction}

In recent years, the explosive user data have been increasing rapidly and numerous related literatures have studied it. The emerging diverse mobile applications include augmented reality (AR), virtual reality (VR), and mobile online gaming [1], which are typically served by a traditional cellular network that equips centralized servers and enable users to enjoy a high QoE [2-4]. However, the traditional cellular networks have difficulties in facing the demands of explosively growing traffic and low-latency transmissions [5-7].

To tackle these problems, mobile edge networks have been proposed to take advantage of the edge caching resources in networks and reduce the distance between contents and UEs [8, 9]. Edge caching and content delivery

${ }^{*}$ Correspondence: luweid@zjut.edu.cn

${ }^{1}$ College of Information Engineering, Zhejiang University of Technology, Liuhe Road, Hangzhou 310023, China

Full list of author information is available at the end of the article are key techniques for a content-centric network [10]. Traditionally, for a cellular network, a user's content request is served by servers located far away from the UE. It will severely block the backhaul links and hence lead to a long delay. Therefore, caching popular contents at the edge of networks such as base stations (BSs) and UEs is helpful to alleviate backhaul load by staggering the peak hours of traffic and obtain a low-latency transmission [11]. The authors of [12] utilized the way of caching at BSs, which contributed to the great benefits for the performance of video capacity and the experience of low latency. In [13], a content placement algorithm together with a resource allocation algorithm was jointly developed to solve the trade-off between the content placement of UEs and resource allocation between UEs and BSs. The results showed that the developed algorithms had outstanding performance in terms of the quality of service guarantee. Notably, it is still unable to meet the demands of lowlatency transmissions when the distance between users and caching devices is large. 
Due to the advantages of high maneuverability, ondemand deployment, and low cost [14], deploying unmanned aerial vehicle (UAVs) over mobile edge networks is becoming a popular research area, which can greatly improve QoE of users in terms of low latency via UAV-UE cooperation content sharing [15-22]. In [16], a block coordinate descent algorithm was developed to solve a joint optimization problem of trajectory and caching in an UAV-enabled mobile edge network, which minimized the energy consumption of the UAV. A novel scheme for UAV-enabled networks was proposed in a content-centric communication system in [19], where the UAVs served the ground users by delivering the requested files and the UEs cached all the files cooperatively. The trade-off between the UAVs and ground users was characterized as an optimization problem, which was solved by jointly designing the file caching strategy, the UAV trajectory, and communication scheduling. The authors of [22] proposed a mean field game approach to solve the distributed delay optimization problem in a multi-UAV edge network. Results demonstrated that the proposed approach significantly reduced the download delay of users. Nevertheless, in reality, due to the privacy concerns and battery life concerns, users are unwilling to cooperation content sharing. Therefore, it is necessary to provide proper rewards with users for encouraging their active content sharing.

Money exchange, resource exchange, and reputation exchange are three widely used incentive mechanism [23-25]. However, these three incentive mechanisms need to assume that UAV can obtain UEs' complete information such as channel conditions, resource requirements, and costs for caching, which is unrealistic for practical implements. Contract theory is an effective method to design incentive mechanisms in a monopoly market under asymmetric information [26-29]. In [27], a contract based on two-layer caching network services was proposed to maximize the profits of network service provider. The authors of [28] devised an optimal contract, which formalized the compatibility of a client with a service and the safe replacement of a service with another service. In [29], the authors developed an incentive contract based on cooperative spectrum sharing, to jointly optimize power and bandwidth distribution.

In this paper, we jointly design an edge caching strategy and reward contract optimization scheme under information asymmetry, to drive the UEs to share more caching resources to improve the social welfare of the UAVenabled mobile edge network, in which the UAV collects the caching resources shared by the ground users. To our best knowledge, this work has not been studied before. The main contributions are as follows:

(1) Giving the models of communication, UAV request, and UE cache, the utility functions of the UAV and UEs as well as the constraints for reward contract feasibility are presented. Taking the heterogeneity of UEs into account, in this paper, three variables related to UEs are considered: UEs' preference towards different contents, UEs' willingness for joining in UAV-UE cooperation content sharing, and transmission delay between the UAV and UEs.

(2) A novel caching algorithm based on the content popularity is developed for acquiring the optimal caching strategy, which can maximize the contents that each UE shares under its cache space limitation. Next, the nonconvex problem is transformed into a solvable one by reducing the constraints. Then, we get the solution via Lagrangian multiplier method. As a result, the optimal reward contract is designed, which maximizes the UAV's utility and ensures the maximum utility of each UE at the same time.

(3) The UE and UAV utility, and the social welfare of the network are used to evaluate the system performance of the proposed scheme. Simulation results show that both the UAV and UEs can benefit from our proposed scheme.

The rest of this paper is organized as follows. The system model and problem formulation are introduced in Section 2. Section 3 designs the optimal caching strategy and reward contract. The simulation results are shown in Section 4. Section 5 draws a conclusion of the paper.

\section{System model and problem formulation}

An UAV-enabled mobile edge network is considered, with one UAV, one BS, and several UEs randomly distributed as demonstrated in Fig. 1 . The set $\mathcal{N}=\{1,2, \ldots, N\}$ denotes the UEs in the network, which can cache popular contents and share with the UAV via UAV-UE links. Taking three variables related to UEs into account: UEs' preference towards different contents, UEs' willingness for joining in UAV-UE cooperation content sharing, and transmission delay between the UAV and UEs. Especially, the UEs' willingness to UAV-UE cooperation content sharing is different from each other, which is one important influencing factor. We assume that UE $i$ provides part of its total capacity to cache contents for sharing, denoted by a certain percentage $\lambda_{i} \in[0,1]$. It is reasonable that a UE who is more actively sharing contents deserves more rewards. Therefore, all the UEs are divided into $N$ different types according to $\lambda_{i}$ in an ascending order: type-1, $\ldots$, type- $i, \ldots$, type- $N$. A UE with higher type means that it will cache and deliver more contents to the UAV via UAV-UE links. Without loss of generality, we assume that $\lambda_{i}$ follows a uniform distribution with probability density function $f(\lambda)=1, \lambda \in[0,1]$, and the cooperative cache percentage $\lambda_{i}$ follows:

$$
\lambda_{1}<\cdots<\lambda_{i}<\cdots<\lambda_{N}, \quad i \in \mathcal{N} .
$$

For a type- $i$ UE, the UAV will reward it with virtual money $a_{i}$ according to the its contribution. For the 


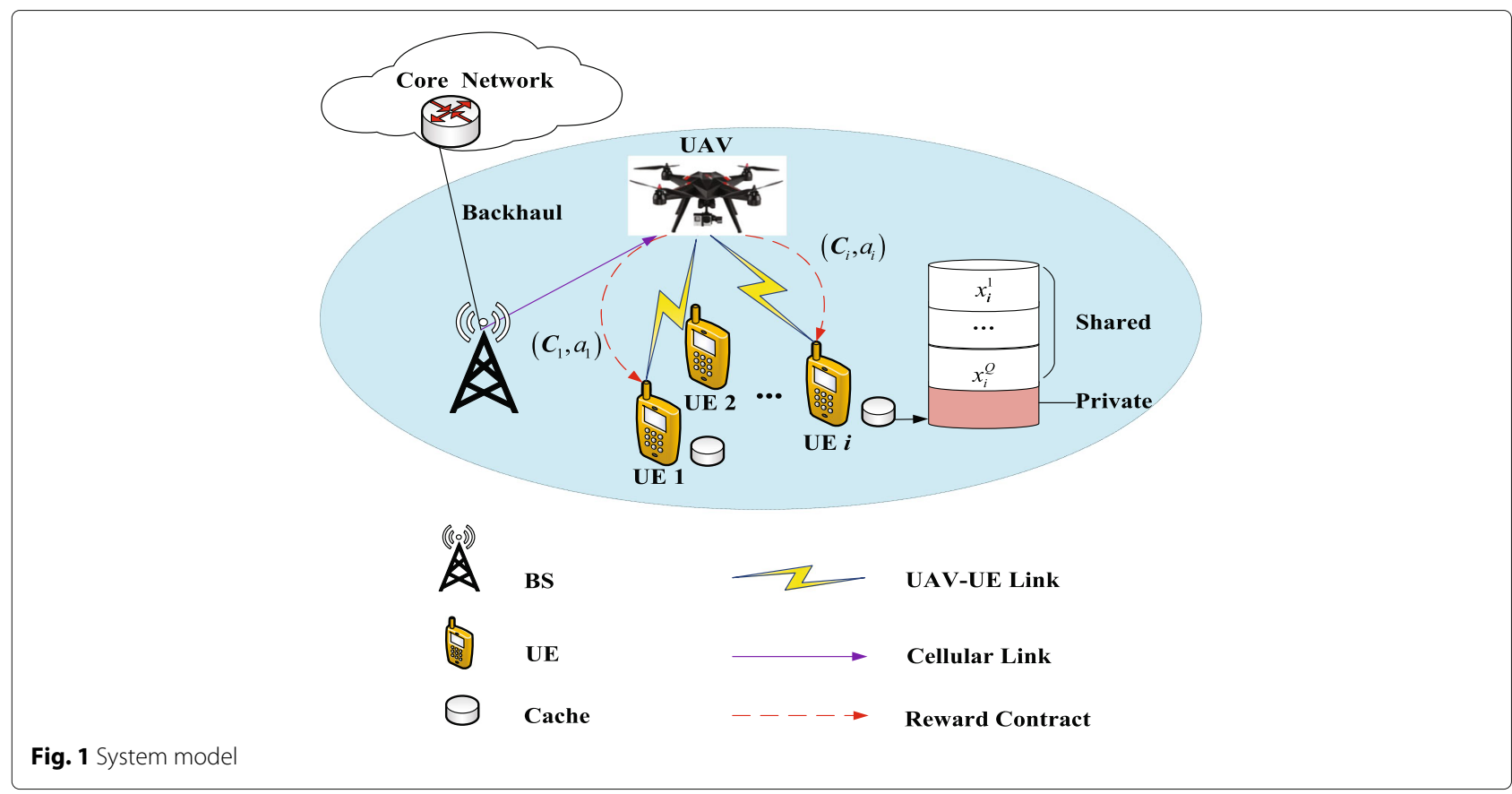

UAV, it will provide a higher reward to a higher type UE. Under information asymmetry, the UAV will design a reward contract based on the UE's contribution and the UAV's reward denoted by $(C, a(C))$, where $C$ represents the total amount of contents that a UE shares and $a$ represents the corresponding reward provided by the UAV. It is obvious that $a(C)$ increases as $C$ since the reasonability that a UE contributes more, deserves more. The UAV can know the probability that a UE belongs to type- $i$ despite the information asymmetry, which can be denoted by $\theta_{i}$ and satisfies $\sum_{i=1}^{N} \theta_{i}=1$. In this paper, we assume that all the UE types have an equal probability with $\theta_{i}=\frac{1}{N}$. The UAV offers a reward contract $\left(C_{i}, a_{i}\right)$ for a type- $i \mathrm{UE}$.

\subsection{Communication model}

In the considered network, these $N$ UEs share their contents with the UAV by using the TDMA technology to improve the social welfare of the network. During a determined finite duration $T$, the UAV hovers in the same air position to request contents from the ground users who are seen as stationary. The finite time $T$ is divided into $K$ time slots, which can be indexed by $t \in \mathcal{T}=\{1,2, \ldots, K\}$.

For simplicity, we establish a three-dimensional (3D) Cartesian coordinate system with the base station as the origin. At time slot $t$, the position coordinates of the BS, the UAV, and UE $i$ are denoted as $(0,0,0)$, $\left(X_{t}, Y_{t}, H_{t}\right)_{t \in \mathcal{T}}$, and $\left(X_{i}^{t}, Y_{i}^{t}, H_{i}^{t}\right)_{i \in \mathcal{N}, t \in \mathcal{T}}$, respectively. Then, the distance between the UAV and UE $i$ is denoted by $d_{i}[t]$

$$
\begin{aligned}
d_{i}[t] & =\sqrt{\left(X_{t}-X_{i}^{t}\right)^{2}+\left(Y_{t}-Y_{i}^{t}\right)^{2}+\left(H_{t}-H_{i}^{t}\right)^{2}}, \\
i & \in \mathcal{N}, t \in \mathcal{T} .
\end{aligned}
$$

Similarly, the distance between the UAV and BS is denoted by

$$
d_{0}[t]=\sqrt{\left(X_{t}\right)^{2}+\left(Y_{t}\right)^{2}+\left(H_{t}\right)^{2}}, \quad t \in \mathcal{T} .
$$

We assume that $d_{0}[t] \gg d_{i}[t]$ for any UE $i$. Therefore, the channel gain between the UAV and UE $i$ at the given time slot $t$ can be described as the free-space path loss model

$$
\begin{aligned}
h_{i}[t] & =\mu_{0} d_{i}^{-2}[t] \\
& =\frac{\mu_{0}}{\left(X_{t}-X_{i}^{t}\right)^{2}+\left(Y_{t}-Y_{i}^{t}\right)^{2}+\left(H_{t}-H_{i}^{t}\right)^{2}}, \\
i & \in \mathcal{N}, t \in \mathcal{T},
\end{aligned}
$$

where $\mu_{0}$ is the channel gain at the reference distance $d_{0}=1 \mathrm{~m}$.

Thus, the transmission rate that UE $i$ delivers contents to the UAV is

$$
R_{i}[t]=B \log _{2}\left(1+\frac{P_{i} h_{i}[t]}{N_{0}}\right), \quad i \in \mathcal{N}, t \in \mathcal{T},
$$

where $B$ represents the transmission bandwidth, $P_{i}$ is the transmission power of $\mathrm{UE} i$, and $N_{0}$ is the channel noise power, respectively. Therefore, the unit transmission delay between the UAV and UE $i$ is 


$$
\begin{aligned}
L_{i}[t] & =\frac{1}{R_{i}[t]} \\
& =\frac{1}{B \log _{2}\left(1+\frac{P_{i} h_{i}[t]}{N_{0}}\right)}, \quad i \in \mathcal{N}, t \in \mathcal{T} .
\end{aligned}
$$

Similarly, the unit service delay that the UAV served by the $\mathrm{BS}$ is expressed as $L_{0}[t]$. It is easy to prove that $L_{0}[t] \gg$ $L_{i}[t]$, for each UE at any time slot.

When a request task starts, the UAV will retrieve the requested content from the neighboring UEs who have cached it in an ascending order according to their unit transmission delay. The index $(i)_{t}$ is used to denote the UE with the $i$ th lowest unit transmission delay to the UAV. The retrieve process will continue as $i$ increases until the UAV receives the complete requested content. If all the UEs' service is still not enough to satisfy the requested content for the UAV, the remaining portion of the content will finally be served by the BS.

\subsection{UAV request model}

Due to the UAV is differently interested in various contents, a heterogeneous request model is considered. We utilize Zipf distribution to model the UAV's request pattern [30]. Thus, the probability of content $q$ with popularity rank $r$ is requested as

$$
\operatorname{Pr}_{r}^{(q)}=\frac{r^{-\beta}}{\sum_{q=1}^{Q} q^{-\beta}}, \quad q \in \mathcal{Q}=\{1,2, \ldots, Q\}, \forall \beta,
$$

where $\mathcal{Q}$ denotes the set of contents, $Q$ denotes the total number of contents, and $\beta$ represents the Zipf distribution exponent. Therefore, the UAV's request heterogeneity is modeled with the randomly generated popularity matrix $\boldsymbol{P} \in \mathbb{R}^{N \times Q}$ of $K$ time slots.

\subsection{UE cache model}

We denote the size of content $q$ as $s_{q}$. All the contents are cached by each UE with a coded caching scheme under a limited cache capacity $Y$ [31]. On this basis, the UEs can cache a certain number of bits of each content. Therefore, a content request of the UAV can be satisfied by nearby UEs via UAV-UE links, if it has obtained a proportion threshold of the complete content in any order. Without loss of generality, we set this proportion threshold to 1 . That means a request task will be successfully finished only when the UAV acquires the complete requested content.

The proportion of content $q$ that UE $i$ caches is denoted by $x_{i}^{q}$, which range of values is $(0,1]$. Thus, we represent the caching strategy of all UEs as an $N \times Q$ matrix $X=\left(x_{i}^{q}\right)_{i \in \mathcal{N}, q \in \mathcal{Q}} \in[0,1]^{N \times Q}$. Moreover, we denote the vector $\left(x_{i}\right)_{i \in \mathcal{N}}$ as the caching state of UE $i$, which has a significant impact on the total amount of contents that UE $i$ shares.

\subsection{UAV-UE cooperation content sharing}

The reward that the UAV offers to a UE depends on its total amount of contents shared to the UAV via UAV-UE links. Due to the total amount of contents shared by a UE are closely relevant to its caching strategy, in order to obtain more rewards, it has to optimize its caching strategy to maximize the total amount of contents shared. At time slot $t$, the proportion of content $q$ shared by UE $i$ to the UAV is denoted as $C_{i, t}^{q}$, which is denoted by

$$
\begin{aligned}
C_{i, t}^{q}(X) & =\min \left\{x_{i}^{q}, \max \left\{0,1-\sum_{k=2}^{[i]_{t}-1} x_{(k)_{t}}^{q}\right\}\right\}, \\
i & \in \mathcal{N}, t \in \mathcal{T}, q \in \mathcal{Q},
\end{aligned}
$$

where $[i]_{t}$ is the ranking of $L_{i}[t]$ in $\left(L_{n}[t]\right)_{n \in \mathcal{N}}$. Thus, the total amount of contents shared by UE $i$ could be computed as follows:

$$
C_{i}(X)=\sum_{q \in \mathcal{Q}} \sum_{t \in \mathcal{T}} s_{\mathrm{q}} C_{i, t}^{q}(X) P_{t}^{q}, \quad i \in \mathcal{N},
$$

where $s_{q}$ and $P_{t}^{q}$ represent the size of content $q$ and the probability of content $q$ requested by the UAV at time slot $t$, respectively. In the following, for convenience, we denote $C_{i}(X)$ as $C_{i}$.

\subsection{Utility functions and social welfare}

When a UE joins in UAV-UE cooperation content sharing, the cost is the total amount of contents it shares to the UAV. And in return, the UAV provides a reward for compensation to drive the UE to cooperate. Thus, the utility function of UE $i$ is defined by the difference between the benefit and the cost when it selects contract $\left(C_{i}, a_{i}\right)$ [32], which can be written as

$$
U_{\mathrm{UE}}(i)=\lambda_{i} V\left(a_{i}\right)-\varepsilon C_{i}, \quad i \in \mathcal{N},
$$

where $V\left(a_{i}\right)$ is the valuation function of reward $a_{i}$ that UE $i$ obtains and $\varepsilon$ represents the UE's unit caching resource cost for sharing. In this paper, we set $\varepsilon=1$. From [33], we can know that $V\left(a_{i}\right)$ is a strictly increasing concave function. Since $V^{\prime}(a)>0$ and $V^{\prime \prime}(a)<0, V\left(a_{i}\right)$ increases as $a_{i}$ and $V\left(a_{i}\right)$ increases slowly when $a_{i}$ becomes large. For simplicity, in this paper, we define $V\left(a_{i}\right)=\delta \ln \left(1+a_{i}\right)$, where $\delta>0$ is a given conversion parameter. Without loss of generality, we set $\delta=2$.

The utility function of the UAV when UE $i$ selects contract $\left(C_{i}, a_{i}\right)$ is defined by the difference between the obtained caching resource and paid reward, which can be written as

$$
U_{\mathrm{UAV}}(i)=C_{i}-\omega a_{i}, \quad i \in \mathcal{N},
$$

where $\omega>0$ is the unit reward cost of the UAV's rewards to the cooperative UEs. For simplicity, we set $\omega=0.015$ in this paper. To benefit from UAV-UE cooperation content 
sharing, the UAV will ensure that $C_{i}-\omega a_{i}>0$. Otherwise, the UAV will turn to the BS for help.

Due to information asymmetry, the UAV only knows the probability that each UE belongs to a certain type denoted by $\theta_{i}$. Thus, the total UAV utility can be given by

$$
U_{\mathrm{UAV}}=\sum_{i=1}^{N} \theta_{i}\left(C_{i}-\omega a_{i}\right), \quad i \in \mathcal{N} .
$$

The overall performance of the system is evaluated by the social welfare, which is defined as the sum of UAV's and UEs' utilities, which can be calculated as

$$
S=\sum_{i=1}^{N}\left[U_{\mathrm{UE}}(i)+U_{\mathrm{UAV}}(i)\right], \quad i \in \mathcal{N} .
$$

\subsection{Reward contract design}

To drive the UEs' active participation in UAV-UE cooperation content sharing, there are several constraints that must be satisfied for the designed contract:

Definition 1 (Individual rationality (IR)) The utility of the contract designed for a type-i UE should be no less than zero:

$$
U_{U E}(i)=\lambda_{i} V\left(a_{i}\right)-C_{i} \geq 0, \quad i \in \mathcal{N} .
$$

Since each UE is at the expense of caching resources, if the reward provided by the UAV is not enough to compensate its paid cost, it will not share its contents for the reason that it cannot benefit from UAV-UE cooperation content sharing.

Definition 2 (Incentive compatibility (IC)) The contract designed specifically for a type-i UE will be most likely selected by UE $i$, which is due to that can achieve its maximum utility.

$$
\lambda_{i} V\left(a_{i}\right)-C_{i} \geq \lambda_{i} V\left(a_{j}\right)-C_{j}, \quad i, j \in \mathcal{N}, i \neq j .
$$

The IC constraints demonstrate that the maximum utility of a type- $i$ UE will be achieved only when the contract $\left(C_{i}, a_{i}\right)$ is selected. Based on this, the UAV can know a UE's type according to the contract selected by the UE.

According to the IR and IC constraints, we have the following Lemma.

Lemma 1 For any UE type contract $\left(C_{i}, a_{i}\right), a_{i}>a_{j}$ if and only if $\lambda_{i}>\lambda_{j}$.

Proof First, the sufficiency will be proved: if $\lambda_{i}>\lambda_{j}$, then $a_{i}>a_{j}$.

From the IC constraints, we can get

$$
\begin{aligned}
\lambda_{i} V\left(a_{i}\right)-C_{i} & \geq \lambda_{i} V\left(a_{j}\right)-C_{j}, \\
\lambda_{j} V\left(a_{j}\right)-C_{j} & \geq \lambda_{j} V\left(a_{i}\right)-C_{i},
\end{aligned}
$$

where $i, j \in \mathcal{N}, i \neq j$. Combining the inequalities (16) with (17), we can get

$$
\lambda_{i} V\left(a_{i}\right)+\lambda_{j} V\left(a_{j}\right) \geq \lambda_{i} V\left(a_{j}\right)+\lambda_{j} V\left(a_{i}\right) .
$$

By merging similar items, we can get

$$
\left(\lambda_{i}-\lambda_{j}\right) V\left(a_{i}\right) \geq\left(\lambda_{i}-\lambda_{j}\right) V\left(a_{j}\right) .
$$

We know that $\lambda_{i}-\lambda_{j}>0$ since $\lambda_{i}>\lambda_{j}$. By dividing both sides of (19) by $\left(\lambda_{i}-\lambda_{j}\right)$, we can get $V\left(a_{i}\right)>V\left(a_{j}\right)$. Since $V\left(a_{i}\right)$ is a strictly increasing function of $a_{i}$, we can derive that $a_{i}>a_{j}$.

Next, the necessity will be proved: if $a_{i}>a_{j}$, then $\lambda_{i}>$ $\lambda_{j}$. Similarly, according to the IC constraints, we can get

$$
\left(V\left(a_{i}\right)-V\left(a_{j}\right)\right) \lambda_{i} \geq\left(V\left(a_{i}\right)-V\left(a_{j}\right)\right) \lambda_{j} .
$$

We can know $V\left(a_{i}\right)-V\left(a_{j}\right)>0$ since $a_{i}>a_{j}$ and $V\left(a_{i}\right)$ is a strictly increasing function of $a_{i}$. Then, dividing both sides of (20) by $\left(V\left(a_{i}\right)-V\left(a_{j}\right)\right)$, we can derive that $\lambda_{i}>\lambda_{j}$.

Lemma 1 shows that a UE will obtain more rewards with a higher type and vice versa. Combining the inequality (1) with Lemma 1, we have the definition as follows:

Definition 3 (Monotonicity) For any UE type contract $\left(C_{i}, a_{i}\right)$, the reward $a_{i}$ follows:

$$
0 \leq a_{1} \leq \cdots \leq a_{i} \leq \cdots \leq a_{N}, \quad i \in \mathcal{N} .
$$

Since $a(C)$ increases as $C$, we can derive the following proposition from Definition 3.

Proposition 1 For any designed contract, the contents shared by the UEs satisfy the following constraint:

$$
0 \leq C_{1} \leq \cdots \leq C_{i} \leq \cdots \leq C_{N}, \quad i \in \mathcal{N} .
$$

Definition 3 and Proposition 1 demonstrate that the more contents a UE shares, the more rewards it can obtain for a feasible contract.

\subsection{Problem formulation}

Under information asymmetry, the objective of the UAV is to maximize its utility by designing the optimal contract. Therefore, the UAV utility maximization problem can be described as

$$
\max _{(X, a)} \sum_{i=1}^{N} \theta_{i}\left[C_{i}(X)-\omega a_{i}\right]
$$

subject to 


$$
\begin{aligned}
& x_{i} \in[0,1]^{1 \times Q}, \\
& \sum_{q \in \mathcal{Q}} x_{i}^{q} s_{q} \leq \lambda_{i} Y, \\
& \lambda_{i} V\left(a_{i}\right)-C_{i} \geq 0 \\
& \lambda_{i} V\left(a_{i}\right)-C_{i} \geq \lambda_{i} V\left(a_{j}\right)-C_{j}, \\
& 0 \leq a_{1} \leq \cdots \leq a_{i} \leq \cdots \leq a_{N}, \\
& i, j \in \mathcal{N}, i \neq j
\end{aligned}
$$

where constraints (24a) are the cache state of $N$ UEs, constraints (24b) are the cache size constraint of $N$ UEs with different types, constraints (24c) represent the IR constraints, constraints (24d) are the IC constraints, and constraint (24e) represents the monotonicity condition. To achieve the maximum utility, all the UEs will optimize their caching strategies to maximize the total amount of contents shared and select the contract specially designed for their own types.

\section{Optimal caching strategy and reward contract}

In this section, we will solve the formulated optimization problem by jointly designing a caching strategy and reward contract scheme. From the above discussion, we can know that a UE with a high utility must have shared large numerous contents. Based on this perception, the optimal caching strategy will be first designed, to maximize the contents shared by each of UE under its cache size constraint. Then, the optimal reward contract will be designed by transforming the problem into a convex optimization problem.

\subsection{Caching strategy}

We can know that the caching strategy of a UE is closely related to the total amount of contents it shares with the UAV according to the discussion in Section 2. We propose a novel caching algorithm based on the content popularity to acquire the optimal caching strategy of each UE. The details are described in Algorithm 1.

The parameters that need to be entered are the total number of UEs $N$, the total number of contents $Q$, the popularity matrix $\boldsymbol{P}$, the delay matrix $D$, the size of content $q$ denoted by $s_{q}$, the total cache space of each UE $Y$, and the collaborative cache percentage of UE $i$ denoted by $\theta_{i}$. Firstly, we initialize the cache state of UEs as zero. Then, based on the popularity matrix of the caching contents, we make the cache percentages that each UE caches from all of the contents have the distribution like as popularity matrix. After obtaining the cache state of UEs, we will calculate $C_{i}$ by using (8) and (9). Finally, the optimal caching strategy is obtained, which makes the contents shared by each of UE reach maximum.

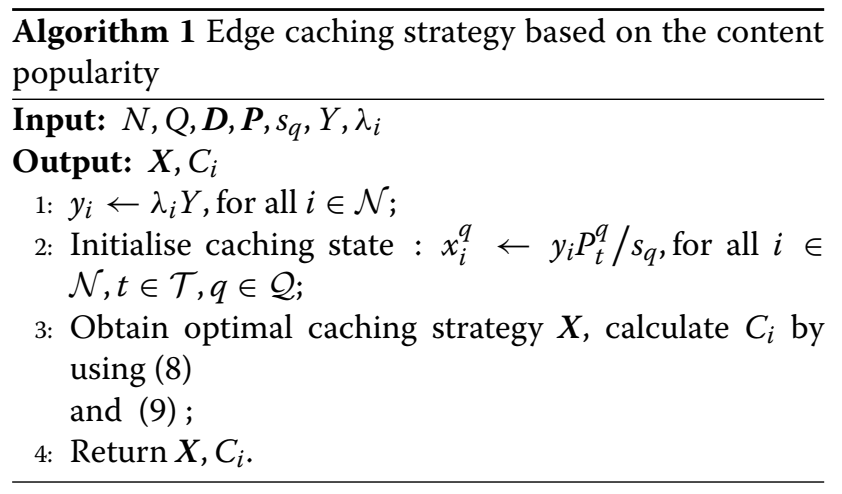

\subsection{Reward contract optimization}

After acquiring the optimal caching strategy, the contract $(X, a)$ can be rewritten as $(C, a)$. The optimization problem (23) can be converted into the problem as follows:

$$
\max _{a} \sum_{i=1}^{N} \theta_{i}\left[C_{i}-\omega a_{i}\right]
$$

subject to

$$
\begin{array}{r}
\lambda_{i} V\left(a_{i}\right)-C_{i} \geq 0, \\
\lambda_{i} V\left(a_{i}\right)-C_{i} \geq \lambda_{i} V\left(a_{j}\right)-C_{j}, \\
0 \leq a_{1} \leq \cdots \leq a_{i} \leq \cdots \leq a_{N}, \\
i, j \in \mathcal{N}, i \neq j .
\end{array}
$$

Next, we will continue to simplify the constraints of the problem in order to solve the problem.

We first simplify IR constraints. According to the constraints (26a), we have a total of $N$ IR constraints. We can know from [32] that these constraints can be reduced to one constraint if the following constraint holds

$$
\lambda_{1} V\left(a_{1}\right)-C_{1} \geq 0 .
$$

Proof According to (1) and IC constraints (26b), we have

$$
\begin{aligned}
\lambda_{i} V\left(a_{i}\right)-C_{i} & \geq \lambda_{i} V\left(a_{1}\right)-C_{1} \\
& \geq \lambda_{1} V\left(a_{1}\right)-C_{1} \geq 0, \quad i \in \mathcal{N} .
\end{aligned}
$$

Thus, we can prove that if the IR constraints are satisfied for type-1 UE, then all of those UE types can be satisfied as well.

Then, we simplify IC constraints. The IC constraints are composed of the downward IC (DIC) and the upward IC (UIC). DIC represents the constraints between type$i$ and type- $j(j \in\{1, \ldots, i-1\})$ UE. In particular, LDIC is the constraint between type- $i$ and type- $(i-1)$ UE. Similar to the DIC and LDIC, the UIC and LUIC represent the constraints between type- $i$ and type- $j(j \in\{i+1, \ldots, N\})$ UE, type- $i$ and type- $(i+1) \mathrm{UE}$, respectively. Then, we can derive the following lemma. 
Lemma 2 For any designed contract, all the DICs will be held if the LDIC is satisfied and all the UICs will be held if the LUIC is satisfied.

Proof We first prove if LDIC holds, then all DICs hold. Considering three UE types that follow $\lambda_{i-1}<\lambda_{i}<\lambda_{i+1}$, we have

$$
\begin{aligned}
& \lambda_{i+1} V\left(a_{i+1}\right)-C_{i+1} \geq \lambda_{i+1} V\left(a_{i}\right)-C_{i}, \\
& \lambda_{i} V\left(a_{i}\right)-C_{i} \geq \lambda_{i} V\left(a_{i-1}\right)-C_{i-1} .
\end{aligned}
$$

Combine (30) with $\lambda_{i}<\lambda_{i+1}$, we can get

$$
\begin{aligned}
\lambda_{i+1}\left[V\left(a_{i}\right)-V\left(a_{i-1}\right)\right] & \geq \lambda_{i}\left[V\left(a_{i}\right)-V\left(a_{i-1}\right)\right] \\
& \geq C_{i}-C_{i-1} .
\end{aligned}
$$

Combine (29) with (31), we have

$$
\begin{aligned}
\lambda_{i+1} V\left(a_{i+1}\right)-C_{i+1} & \geq \lambda_{i+1} V\left(a_{i}\right)-C_{i} \\
& \geq \lambda_{i+1} V\left(a_{i-1}\right)-C_{i-1} .
\end{aligned}
$$

Thus, we can get

$$
\lambda_{i+1} V\left(a_{i+1}\right)-C_{i+1} \geq \lambda_{i+1} V\left(a_{i-1}\right)-C_{i-1} .
$$

Thus, we have proved that if the LDIC for type- $i$ UE holds, then the IC constraint for type- $(i-1)$ UE holds as well. The process can be extended downward from type-( $i-$ 1) to type-1 UEs, which can prove that all DICs hold as follows:

$$
\begin{aligned}
\lambda_{i+1} V\left(a_{i+1}\right)-C_{i+1} & \geq \lambda_{i+1} V\left(a_{i-1}\right)-C_{i-1} \\
& \geq \cdots \\
& \geq \lambda_{i+1} V\left(a_{1}\right)-C_{1} \\
i & =1,2, \ldots, N-1 .
\end{aligned}
$$

Similar to the proof of LDIC, we prove that all UICs will be held if LUIC is satisfied. Considering the same three UE types, we can get

$$
\begin{aligned}
& \lambda_{i-1} V\left(a_{i-1}\right)-C_{i-1} \geq \lambda_{i-1} V\left(a_{i}\right)-C_{i}, \\
& \lambda_{i} V\left(a_{i}\right)-C_{i} \geq \lambda_{i} V\left(a_{i+1}\right)-C_{i+1} .
\end{aligned}
$$

Combine (36) with $\lambda_{i}>\lambda_{i-1}$, we can get

$$
\begin{aligned}
C_{i+1}-C_{i} & \geq \lambda_{i}\left[V\left(a_{i+1}\right)-V\left(a_{i}\right)\right] \\
& \geq \lambda_{i-1}\left[V\left(a_{i+1}\right)-V\left(a_{i}\right)\right] .
\end{aligned}
$$

Combine (35) with (37), we have

$$
\begin{aligned}
\lambda_{i-1} V\left(a_{i-1}\right)-C_{i-1} & \geq \lambda_{i-1} V\left(a_{i}\right)-C_{i} \\
& \geq \lambda_{i-1} V\left(a_{i+1}\right)-C_{i+1} .
\end{aligned}
$$

Thus, we have

$$
\lambda_{i-1} V\left(a_{i-1}\right)-C_{i-1} \geq \lambda_{i-1} V\left(a_{i+1}\right)-C_{i+1} .
$$

Thus, we also have proved that if the LUIC for type- $(i-1)$ UE holds, then the IC constraint for type- $i$ UE holds as well. The process can be extended upward from type- $(i+$
1) to type- $N$ UEs, which can prove that all UICs hold as follows:

$$
\begin{aligned}
\lambda_{i-1} V\left(a_{i-1}\right)-C_{i-1} & \geq \lambda_{i-1} V\left(a_{i+1}\right)-C_{i+1} \\
& \geq \cdots \\
& \geq \lambda_{i-1} V\left(a_{N}\right)-C_{N} \\
& i=2, \ldots, N .
\end{aligned}
$$

The proof is accomplished.

At last, due to the equivalence of LDIC and LUIC, we only consider the constraints as LDIC in the following simplification. Therefore, all the IC constraints could be simplified given that the LDIC holds. After the above simplification is completed, the problem (25) can be simplified as

$$
\max _{a} \sum_{i=1}^{N} \theta_{i}\left[C_{i}-\omega a_{i}\right]
$$

subject to

$$
\begin{aligned}
& \lambda_{1} V\left(a_{1}\right)-C_{1}=0, \\
& \lambda_{i} V\left(a_{i}\right)-C_{i}=\lambda_{i} V\left(a_{i-1}\right)-C_{i-1}, \\
& 0 \leq a_{1} \leq \cdots \leq a_{i} \leq \cdots \leq a_{N},
\end{aligned}
$$

$$
i=1,2, \ldots, N \text {. }
$$

As we can see, the main simplification in this section is to simplify the two inequality constraints of IR and IC. If we ignore the monotonicity condition (42c), it is a standard convex optimization problem, which can be solved by the Lagrangian multiplier method.

To solve the problem (41), we construct the Lagrangian function as follows:

$$
\begin{aligned}
& L\left(a_{i}, \gamma_{1}, \gamma_{2}\right) \\
& \quad=\sum_{i=1}^{N} \theta_{i}\left[C_{i}-\omega a_{i}\right]+\gamma_{1}\left[\lambda_{1} V\left(a_{1}\right)-C_{1}\right]+ \\
& \quad \gamma_{2}\left[\lambda_{i} V\left(a_{i}\right)-C_{i}-\lambda_{i} V\left(a_{i-1}\right)+C_{i-1}\right] \\
& \quad=F+\gamma_{1}\left[\lambda_{1} V\left(a_{1}\right)-C_{1}\right]-\gamma_{2}\left[C_{i}-C_{i-1}\right], \\
& i=1,2, \ldots, N,
\end{aligned}
$$

where $F=\sum_{i=1}^{N} \theta_{i}\left[C_{i}-\omega a_{i}\right]+\gamma_{2} \lambda_{i}\left[V\left(a_{i}\right)-V\left(a_{i-1}\right)\right]$, $V\left(a_{i}\right)=\delta \ln \left(1+a_{i}\right), \delta=2$ and $\omega=2$.

Then let

$$
\begin{aligned}
& \frac{\partial L}{\partial a_{i}}=\frac{\partial F}{\partial a_{i}}=-\omega+\frac{\gamma_{2} \lambda_{i} \delta}{1+a_{i}}=0, \\
& \frac{\partial L}{\partial \gamma_{1}}=\lambda_{1} V\left(a_{1}\right)-C_{1}=0,
\end{aligned}
$$

we can get 


$$
\begin{aligned}
& a_{i}=\frac{\gamma_{2} \delta \lambda_{i}}{\omega \theta_{i}}-1, \\
& \lambda_{1} \delta \ln \left(1+a_{1}\right)-C_{1}=0,
\end{aligned}
$$

thus,

$$
a_{i}=\frac{\lambda_{i} e^{\frac{C_{1}}{\delta \lambda_{1}}}}{\lambda_{1}}-1,
$$

We can find from (48) that the solution of the optimization problem satisfies the monotonicity condition (42c), and hence, it is the optimal solution. We denote the optimal solution as $a_{i}^{*}$, which can be expressed as

$$
a_{i}^{*}=\frac{\lambda_{i} e^{\frac{C_{1}}{\delta \lambda_{1}}}}{\lambda_{1}}-1, \quad i \in \mathcal{N} .
$$

\section{Simulation results and discussions}

In the simulation, we consider a simple UAV-enabled mobile edge network with $N=6$ UEs , $Q=80$ contents. There are different types of UE, which has a total capacity of $Y=20 \mathrm{Mbit}$. Each content has the same size with $s_{q}=5 \mathrm{Mbit}$. The probability that each UE belongs to a certain type is equal to $\theta_{i}=\frac{1}{N}$. The unit resource cost and unit reward cost are set to $\varepsilon=1$ and $\omega=0.015$, respectively. The Zipf parameter is set to $\beta=0.8$. The channel power gain is assumed to be $\mu_{0}=-60 \mathrm{~dB}$ at a reference distance $d_{0}=1 \mathrm{~m}$. The receiver noise power is set to be $N_{0}=-70 \mathrm{dBm}$, and the transmission bandwidth is set to be $B=1 \mathrm{MHz}$. The maximum transmission power of the UEs is equal to $P_{i}=20 \mathrm{dBm}$. The cache size equals to $12 \%$. In addition, the performance of the proposed caching strategy is compared with two benchmark schemes, fair caching and random caching, where the fair caching scheme is that each UE's caching proportion is the same for all the contents, while the random caching scheme is that each UE randomly caches the contents in the network until full with its cache space.

Figure 2 shows the total amount of contents shared versus the UE type. In Fig. 2, we can find that the total amount of contents increase as the UE type, which satisfies the monotonicity condition of Proposition 1 . We can also observe that the contents the UEs share with the fair caching strategy are less than our proposed strategy, but more than the random caching strategy, which indicates that our proposed caching strategy can make more efficient use of edge caching resources. Furthermore, we can see that the higher the UE type is, the more obvious the advantage of our proposed caching strategy has than the other two strategies.

Figure 3 shows the rewards provided by the UAV versus the UE type. It is obvious to find that the rewards increase as the UE type in Fig. 3, which satisfies the monotonicity condition of Definition 3. Similarly, we can also find from

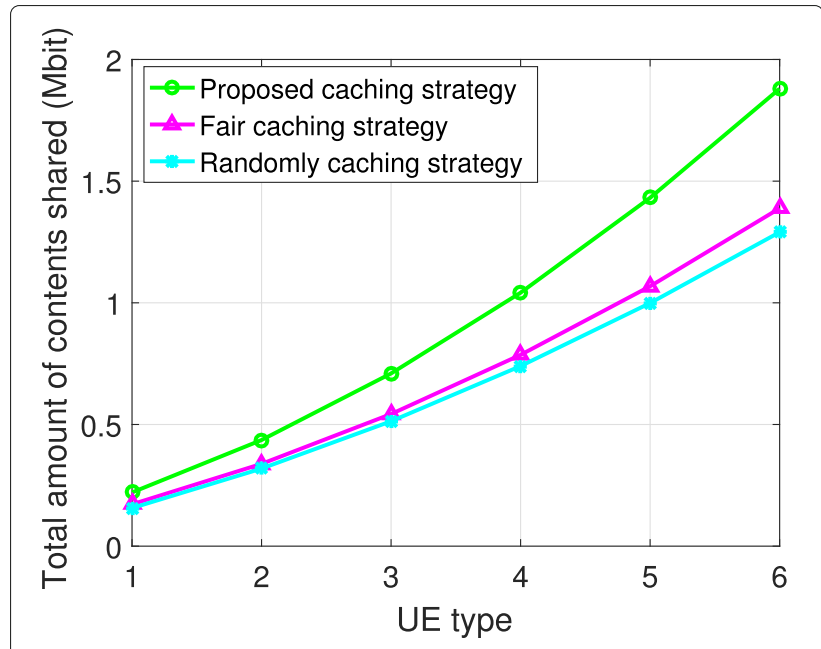

Fig. 2 Total amount of contents shared versus the UE type

Fig. 3 that each type of UE will obtain the largest reward with the proposed caching strategy, followed by the fair caching strategy, while obtaining the least reward with the randomly caching strategy. And the difference among the three strategies will grow larger with higher UE types.

Figure 4 shows the UE and UAV utility, and social welfare with the cache sizes equal to $10 \%$ and $15 \%$ versus the UE type, where cache size means the percentage of total cache that the UEs is willing to share to the total content size. In Fig. 4, we can see that the higher the UE type is, the larger utility will obtain for the UAV, UEs, and social welfare of the UAV-enabled mobile edge network, which complies with the monotonicity. We first compare the impact of different UE types on system performance. As the UE type increases, the total utilities of the UAV and UEs, and social welfare will also increase. We can also find that the utilities of the UEs increase faster than the UAV's,

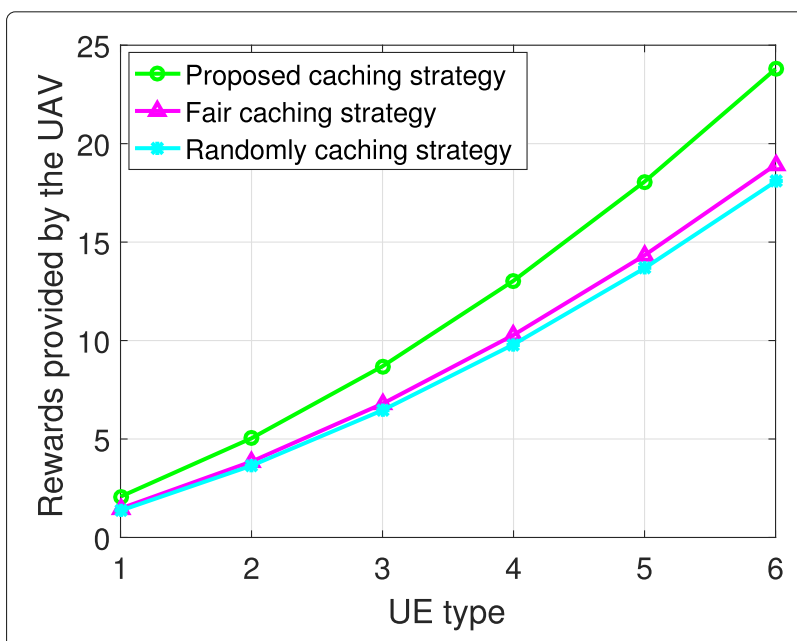

Fig. 3 Rewards provided by the UAV versus the UE type 


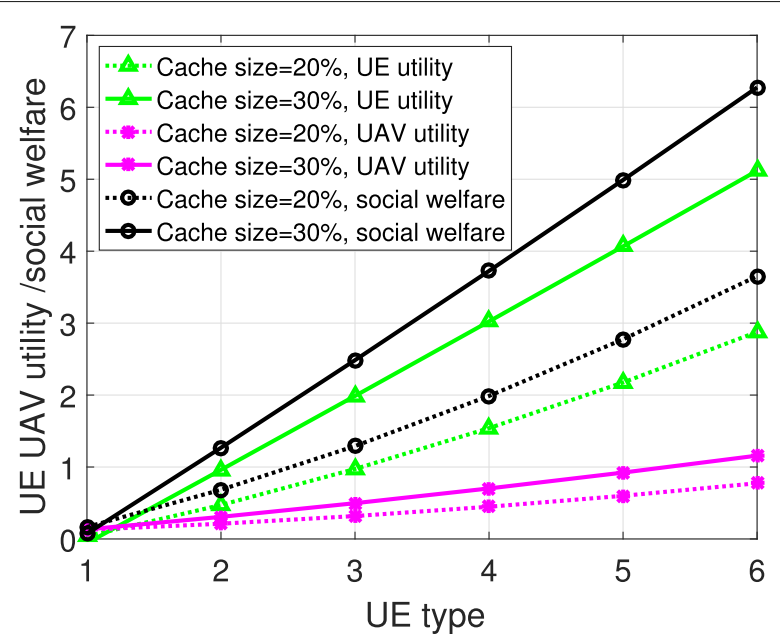

Fig. $4 \cup E$ and UAV utility, and social welfare versus the UE type

which is due to the limited contents shared by the UEs and their utility functions. Then, we compare the impact of different cache sizes on system performance. We can see that the UE and UAV utility, and social welfare with the cache size equals to $15 \%$ perform better than it when the cache size equals to $10 \%$, which indicates that when the UE type is determined, the larger the cache size is, the higher utility it will achieve. Since both of the UAV and UEs gain a non-negative utility, the social welfare of the network also obtains a non-negative utility, which is a win-win situation.

Figure 5 shows the UE utility with three different UE types versus the contract designed for UE type- $i$. In Fig. 5, we can see that each of UE type will achieve maximal utility when it selects the contract specially designed for its own type, which indicates that our designed contract satisfies the IC constraints. For instance, the type-5 UE

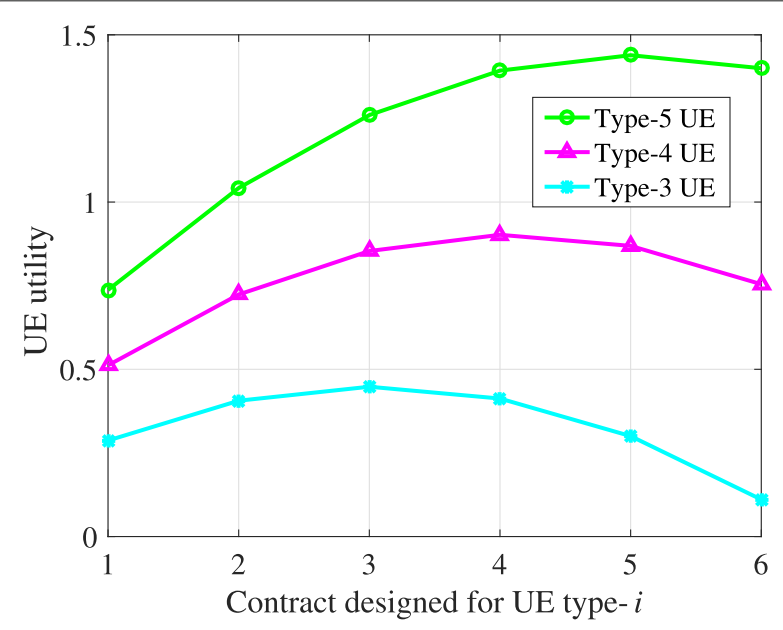

Fig. 5 UE utility versus the contract designed for UE type- $i$

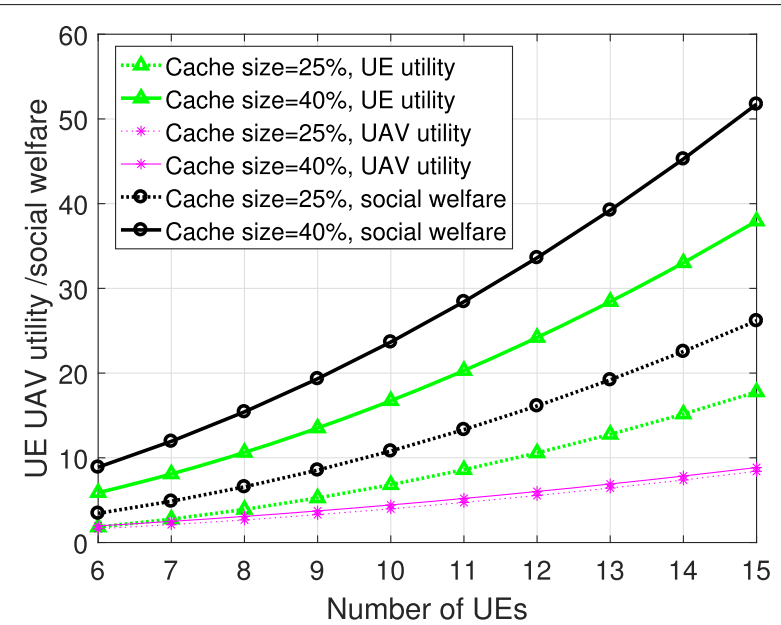

Fig. 6 UE and UAV utility, and social welfare versus the cache size and number of UES

will obtain maximal utility when it selects the contract designed for type- 5 UE. We can also observe from Fig. 5 that with a same contract, the utility of type-5 UE is larger than the other two UE types, which indicates that the higher the UE type is, the higher utility it will obtain.

Figure 6 shows the UE and UAV utility, and social welfare with the cache sizes equal to $25 \%$ and $40 \%$ versus the number of UEs. We first compare the impact of different total UE numbers on system performance. In Fig. 6, we can find that as the number of UEs increases, the total utilities of the UAV and UEs, and social welfare will also increase. This is exactly one of the important advantages of the UAV-enabled mobile edge network that allows the system to take advantage of plenty of edge caching resources. Then, we compare the impact of different cache sizes on system performance. When the cache size equals to $25 \%$, we can see that the utilities perform worse than the case when the cache size equals to $40 \%$, which indicates that when the number of UEs are determined, the larger the cache size is, the larger utility it will achieve. That is to say, with more number of UEs and larger total cache size, it will contribute more to the whole network.

\section{Conclusions}

In this paper, we jointly design an edge caching strategy and reward contract optimization scheme for the UAV-enabled mobile edge network, aiming to motivate the users actively joining in UAV-UE cooperation content sharing. We formulate and jointly design an edge caching strategy and reward contract optimization problem to maximize the UAV's utility. Based on the content popularity, we propose a novel edge caching algorithm. The feasibility of the optimal reward contract is proved by theoretical and numerical analysis. Numerical results show the efficiency of our proposed scheme. 


\section{Abbreviations}

AR: Augmented reality; BS: Base station; DIC: Downward incentive compatibility; IC: Incentive compatibility; IR: Individual rationality; LDIC: Local downward incentive compatibility; LUIC: Local upward incentive compatibility; QoE: Quality of experience; UAV: Unmanned aerial vehicle; UE: User equipment; UIC: Upward incentive compatibility; VR: Virtual reality

\section{Acknowledgements}

This work was supported by the National Natural Science Foundation of China under grant 61871348 .

\section{Authors' contributions}

WL and BY conceived and designed the corresponding system model. GH optimized the proposed models. BY and BL performed the simulations of the model. WL and BY wrote the paper. All authors read and approved the final manuscript.

\section{Funding}

National Natural Science Foundation of China under grant 61871348

\section{Availability of data and materials}

Data sharing is not applicable to this article as no datasets were generated or analyzed during the current study.

\section{Competing interests}

The authors declare that they have no competing interests.

\section{Author details}

${ }^{1}$ College of Information Engineering, Zhejiang University of Technology, Liuhe Road, Hangzhou 310023, China. ${ }^{2}$ School of Information and Electrical Engineering, Harbin Institute of Technology, Weihai 264209, China.

Received: 4 December 2019 Accepted: 26 January 2020

Published online: 10 February 2020

\section{References}

1. F. Zhou, Y. Wu, R. Hu, et al., Computation rate maximization in UAV-enabled wireless-powered mobile-edge computing systems. IEEE J. Sel. Areas Commun. 36(9), 1927-1941 (2018)

2. Y. Cao, N. Zhao, F. Yu, et al., Optimization or alignment: secure primary transmission assisted by secondary networks. IEEE Journal Sel. Commun. 36(4), 905-917 (2018)

3. W. Lu, Y. Gong, X. Liu, et al., Collaborative energy and information transfer in green wireless sensor networks for smart cities. IEEE Trans. Indus. Infor. 14(4), 1585-1593 (2018)

4. X. Liu, X. Zhai, W. Lu, et al., QoS-guarantee resource allocation for multibeam satellite industrial internet of things with NOMA. IEEE Trans. Indus. Infor. (2019). https://doi.org/10.1109/tii.2019.2951728

5. N. Zhao, N. Golrezaei, A. G. Dimakis, et al., Communications, caching, and computing oriented small cell networks with interference alignment. IEEE Commun. Mag. 54(9), 29-35 (2016)

6. Z. Na, X. Liu, F. Yu, et al., Subcarrier allocation based simultaneous wireless information and power transfer algorithm in $5 \mathrm{G}$ cooperative OFDM communication systems. Phys. Commun. 29, 164-170 (2018)

7. W. Lu, Y. Zhang, M. Wang, et al., Cooperative spectrum sharing in OFDM two-way relay systems with bidirectional transmissions. IEEE Commun. Lett. 21(6), 1349-1352 (2017)

8. N. B. Hassine, P. Minet, D. Marinca, et al., Popularity prediction-based caching in content delivery networks. Ann. Telecommun. 74(2), 351-364 (2019)

9. Z. Zhang, Y. Yang, M. Hua, et al., Proactive caching for vehicular multi-view 3D video streaming via deep reinforcement learning. IEEE Trans. Wire. Commun. 18(5), 2693-2706 (2019)

10. S. Wang, X. Zhang, L. Wang, et al., Joint design of device to device caching strategy and incentive scheme in mobile edge networks. IET Commun. 12(14), 1728-1736 (2018)

11. C. Xu, X. Wang, Transient content caching and updating with modified harmony search for Internet of Things. Digital Commun. Netw. 5(1), 24-33 (2019)

12. H. Ahlehagh, S. Dey, Video-aware scheduling and caching in the radio access network. IEEE/ACM Trans. Netw. 22(5), 1444-1462 (2014)
13. W. Jing, X. Wen, Z. Lu, et al., Multi-location-aware joint optimization of content aching and delivery for backhaul-constrained UDN. Sensors. 19(11), 2449-2469 (2019)

14. Y. Zeng, R. Zhang, T. J. Lim, Wireless communications with unmanned aerial vehicles: opportunities and challenges. IEEE Commun. Mag. 54(5), 36-42 (2016)

15. M. Hua, Y. Wang, C. Li, et al., UAV-aided mobile edge computing systems with one by one access scheme. IEEE Trans. Green Commun. Net. 3(3), 664-678 (2019)

16. H. Mei, K. Wang, D. Zhou, et al., Joint trajectory-task-cache optimization in UAV-enabled mobile edge networks for cyber-physical system. IEEE Access. 7, 156476-156488 (2019)

17. B. Jiang, J. Yang, H. Xu, et al., Multimedia data throughput maximization in Internet-of-Things system based on optimization of cache-enabled UAV. IEEE Internet Things J. 6(2), 3525-3532 (2019)

18. F. Cheng, N. Gui, N. Zhao, et al., UAV-relaying-assisted secure transmission with caching. IEEE Trans. Commun. 67(5), 3140-3153 (2019)

19. X. Xu, Y. Zeng, Y. Guan, et al., Overcoming endurance issue: UAV-enabled communications with proactive caching. IEEE J. Sel. Areas Commun. 36(6), 1231-1244 (2018)

20. N. Zhao, W. Lu, M. Sheng, et al., UAV-assisted emergency networks in disasters. IEEE Wirel. Commun. 26(1), 45-51 (2019)

21. N. Zhao, F. Cheng, F. Yu, et al., Caching UAV assisted secure transmission in hyper-dense networks based on interference alignment. IEEE Trans. Commun. 66(5), 2281-2294 (2018)

22. K. Xue, L. Li, F. Yang, et al., in 201928 th Wireless and Optical Communications Conference (WOCC). Multi-UAV delay optimization in edge caching networks: a mean field game approach (IEEE, pp. 1-5. https://doi.org/10.1109/wocc.2019.8770639

23. J. Zhou, C. Li, C. Zhai, et al., Joint pricing and cache placement for video caching: a game theoretic approach. IEEE J. Sel. Areas Commun. 37(7), 1566-1583 (2019)

24. M. Tao, Y. Liu, Spectrum leasing and cooperative resource allocation in cognitive OFDMA networks. J. Commun. Netw. 15(1), 102-110 (2013)

25. C. Wang, G. Lin, C. Chou, et al., Device-to-device communication in LTE-advanced system: a strategy-proof resource exchange framework. IEEE Trans. Veh. Technol. 65(12), 10022-10036 (2016)

26. Y. Zhang, M. Pan, L. Song, et al., A survey of contract theory-based incentive mechanism design in wireless networks. IEEE Wirel. Commun. 24(3), 80-85 (2017)

27. T. Liu, J. Li, F. Shu, et al., Incentive mechanism design for two-layer wireless edge caching networks using contract theory. IEEE Trans. Serv. Comput., 1-12 (2018). https://doi.org/10.1109/TSC.2018.2882842

28. G. Castagna, N. Gesbert, L. Padovani, A theory of contracts for web services. ACM Trans. Program. Lang. Syst. 31(5), 261-272 (2009)

29. W. Lu, S. Hu, X. Liu, et al., Incentive mechanism based cooperative spectrum sharing for OFDM cognitive loT network. IEEE Trans. Netw. Sci. Eng. (2019). https://doi.org/10.1109/TNSE.2019.2917071

30. Q. Li, Y. Zhang, X. Ge, et al., D2D-assisted caching on truncated Zipf distribution. IEEE Access. 7, 13411-13421 (2019)

31. Z. Chen, Y. Liu, B. Zhou, et al., in 2016 IEEE International Conference on Communications (ICC). Caching incentive design in wireless D2D networks: a Stackelberg game approach IEEE, 2016). https://doi.org/10. 1109/icc.2016.7511284

32. S. Hong, C. Wernz, J. D. Stillinger, Optimizing maintenance service contracts through mechanism design theory. Appl. Math. Model. 40(21-22), 8849-8861 (2016)

33. Y. Zhang, L. Song, W. Saad, et al., Contract-based incentive mechanisms for device-to-device communications in cellular networks. IEEE J. Sel. Areas Commun. 33(10), 2144-2155 (2015)

\section{Publisher's Note}

Springer Nature remains neutral with regard to jurisdictional claims in published maps and institutional affiliations. 